\title{
Traduzione, fedeltà e riscrittura in alcune traduzioni della Divina Commedia in lingua portoghese
}

Prof. Guido Alberto Bonomini Lingua e Letteratura Italiana UFF.

Resumo: Embora seja lícita a modulação como estratégia na tradução a fim de produzir, na língua de chegada, algumas expressões idiomáticas próprias da língua de partida, ocorre com frequência em algumas traduções, especialmente de poesia e particularmente da poesia de Dante, que o tradutor deseje substituir o autor e acabe por criar um novo texto-contexto poético. Todavia, sabemos que, muito embora as traduções jamais sejam o original e constituam um novo texto, seria auspicioso fazer com que o texto se aproximasse o máximo possível do texto de partida, conservando uma certa credibilidade filológica do produto final, isto é, a vontade do autor.

Palavras-chave: Tradução. Divina Comédia. Texto. Contexto Poético.

Abstract: Malgrado sia lecita la modulazione, come strategia nella traduzione, per rendere nella lingua di arrivo alcune particolari frasi, spesso idiomatiche della lingua di partenza, accade spesso in alcune traduzioni soprattutto di poesia e in particolare nella Divina Commedia di Dante, in alcune traduzioni in lingua portoghese, che il traduttore si voglia sostituire all'autore, ricreando un nuovo testo-contesto poetico. Sappiamo, tuttavia, che nelle traduzioni, malgrado queste non siano mai l'originale, anzi rappresentino sempre e comunque un nuovo testo, sarebbe auspicabile lasciare, quando possibile, il testo tradotto il più possibile letteralmente vicino al testo di partenza, mantenendo una certa credibilità filologica del prodotto finale, ossia, la volontà dell'autore.

Keywords: Traduzione. Divina Commedia. Testo. Contesto Poetico.

Insegnare letteratura italiana all'estero non è un impresa agevole, e, soprattutto quando l'autore è Dante Alighieri. Spesso i miei alunni chiedono se è possibile consultare una traduzione della Divina Commedia, per facilitare la Revista Italiano UERJ 
comprensione testuale, spesso assai complessa e, fino ad ora non gli ho saputo rispondere opportunamente. La domanda a questo punto è: esiste una traduzione che possa facilitare la comprensione, l'esegesi, la didattica della Divina Commedia? Forse no, se nella traduzione speriamo di ritrovare il testo di Dante tradotto letteralmente in portoghese, forse si, se le edizioni delle traduzioni possono venir usate con la dovuta, preliminare cautela. Non è forse qui il luogo per gettare la croce addosso a traduzioni e traduttori che si sono cimentati in un impresa insormontabile come questa, ma vorrei comunque e sempre riaffermare che, come punto di partenza, la traduzione deve, sempre e comunque rispettare la voce dell'autore, non già quella del traduttore che abbia velleità di autore. Sarebbe oltremodo difficile mettere a confronto le innumerevoli edizioni presenti sul mercato brasiliano, mi limiterò qui soltanto a una semplice premessa, come quelle che spesso introducono alcune traduzioni della Divina Commedia in portoghese. Innanzitutto traduzione in versi o in prosa? Per quale optare se non si riesce a penetrare il senso dei versi? Per fini didattici, risponderei subito, sarebbe preferibile una versione in prosa, anche perché spesso, come è tendenza generale di quelle in versi, i traduttori nel tentativo di mantenere la sonorità dei versi, travisano il senso primo del testo di partenza, e quindi, per l'incauto studente di letteratura italiana in Brasile sono assolutamente inservibili; a nulla serve infatti perdersi dietro gli artifici dei traduttori per poter cercare di capire i versi di Dante, si rischia di leggere tutto un altro testo. Temo che questa moda di mantenere la sonorità del verso, stravolgendo completamente il testo, sia una tendenza inaugurata proprio dal nostro poeta Giuseppe Ungaretti, professore di letteratura italiana a San Paolo negli anni trenta del secolo scorso. Ma forse non è lui il responsabile!Traduzioni poetiche fatte da un poeta, risultano essere nulla di più lontano dal testo di partenza. A questa si aggiunga la tendenza creativa di molti traduttori brasiliani e portoghesi e il gioco è fatto: abbiamo davanti agli occhi un altro testo che solo lontanamente ricorda il testo di Dante. E' per questo che a puro titolo informativo, propongo qui 
sotto, alcuni saggi di traduzione del IV canto dell' Inferno di Dante, solo per mostrare con quali strategie di traduzione viene reso il testo di arrivo.

Una delle traduzioni più elogiate anche tra $\mathrm{i}$ siti internet è proprio quella di Cristiano Martins dell' Edizione Itatiaia, Belo Horizonte, 1999, la cui seconda edizione aggiornata è del 2007. A mio avviso non appare encomiabile affatto, essa presenta infatti numerose ricreazioni testuali che poco rendono il senso del poeta fiorentino. Vediamo ora le prime terzine del IV Canto dell'Inferno a confronto:

O sono que alienou meu pensamento a um trovão dissipou-se, e fui tornando à vida de que a mente é o fundamento.
Ruppemi l'alto sonno nella testa un greve truono, si ch'io mi riscossi Come persona che è per forza desta;

Appare evidente che, nel primo verso, come esempio, il traduttore abbia tradotto fedelmente un'unica parola: sonno, il resto è una pura interpretazione e anche di pessimo gusto artistico. Infatti il pensiero/ pensamento, non è presente nel testo dantesco, così come anche nel secondo, l'unica parola fedelmente tradotta è tuono/trovão. Nel terzo verso poi, la bella similitudine dantesca, quotidianissima, della persona svegliata di soprassalto da un rumore improvviso, è resa con uno pseudo ragionamento filosofico sulla mente che è fondamento della vita, paradigma del tutto assente nel testo dantesco. Ma andiamo oltre anche per cercare di capire almeno la strategia di traduzione.

A vista descansada então lançando e l'occhio riposato intorno mossi, em derredor, olhei detidamente, dritto levato, e fiso riguardai a ver onde me achava, e como, e quando. Per conoscer lo loco dov'io fossi.

Nel terzo verso tradotto appare evidente che il traduttore si atteggi a novello Dante, dal momento che introduce il como e quando, /come e quando, tipico intercalare di gusto dantesco, ma non presente in questo passaggio. Si potrebbe dire quindi che questo testo, se si vuol leggere Dante, non serve, visto che il traduttore 
si è talmente immedesimato nell'autentico autore, da non distinguere quando traduce o crea di sana pianta, senza esplicita necessità.

Lo stesso passo tradotto da Xavier Pinheiro nella storica traduzione del 1915, edita da Jacinto Ribeiro dos Santos a Rio de Janeiro, è cosi reso, vediamolo:

\author{
Desse profundo sono fui tirado \\ por horrido estampido estremecendo \\ como quem é por força despertado. \\ Ergui-me, e, os olhos quietos ja volvendo \\ Perscruto por saber onde me achava, \\ e tudo no lugar sinistro atendo.
}

Anche qui la traduzione non è certamente letterale ma, almeno tende a riprodurre le fasi salienti della terzina. In primo luogo il senso del testo di partenza è conservato, l'esser desto da un rumore improvviso, che qui non è un tuono, anche se la chiusa della terzina tradotta, riproduce la similitudine di Dante. Il tradurre di Xavier Pinheiro, che tende a mantenere il ritmo dantesco, ritorna comunque al testo in alcuni punti chiave, anche se a il traduttore tende a interpretare alcuni passaggi, come per esempio la chiusa della seconda terzina in analisi: Dante non riafferma l'idea del luogo sinistro/ lugar sinistro, è una ricreazione del traduttore che, in questo caso tende a sostituirsi all'autore. Sappiamo che in una traduzione, per ovviare a forme idiomatiche o intraducibili parallelamente nella lingua di arrivo, il portoghese in questo caso, sono permesse delle modulazioni che possano rendere lo stesso contesto. Purtroppo nelle traduzioni in versi, vediamo che questo stratagemma va spesso oltre, il testo tradotto diviene così assai spesso sovrabbondante, aggiungendo elementi nuovi non presenti nel testo di partenza.

Lo stesso passo del canto IV dell' Inferno tradotto in prosa da Hernani Donato per la Editora Cultrix, forse senza abbandonarsi a pretese poetiche da parte del traduttore, tende a rendere giustizia al testo del poeta:

Violento estrondo interrompeu aquele torpor profundo, despertando-me qual homem que em sobressalto acorda. Movi o olhar pelo que havia ao meu redor, 
ao longe e perto. De pé me ergui, procurando conhecer o lugar onde me incontrava.

Anche qui si possono notare delle interpretazioni, come nel caso del violento estrondo per il tuono, il torpor profundo che, comunque, tende a interpretare il sonno ne la testa, ma anche qui la chiusa della terzina, la similitudine, è resa con una parallela traduzione in lingua portoghese. Nel verso che segue la prima terzina, il traduttore riproduce con un buon parallelismo l'atto di guardarsi intorno del poeta per capire dove si trovi, alzandosi in piedi. Certo, questa traduzione non è impreziosita dal tentativo di riprodurre la versificazione in terzine di endecasillabi, ma a mio vedere, potrebbe essere una valida o quantomeno simile base didattica, per poter captare il testo tradotto dalla lingua italiana. A questo proposito torna utile ripercorrere il saggio del prof. Mario Vilela dell' Univercidade do Porto nel suo saggio intitolato, Nel mezzo del cammin, ( actas da jornada de estudos italianos em honra de Giuseppe Mea), dove sono messe a confronto, anche lì, tre traduzioni tra le più riconosciute anche in Portogallo. Tra queste, di nuovo, quella di Pedro Xavier Pinheiro del 1915, edita da Jacinto Ribeiro dos Santos, Rio de Janeiro, quella di Marques Braga edita per la prima volta a Lisbona nel 1955 (ma quella usata dal nostro è del 1994) e per concludere quella di Vasco Graça Moura, edita da Bertrand nel 1995. Penso che questo saggio sia altamente esplicativo per capire i gusti e le tendenze delle traduzioni, soprattutto quelle storiche della Divina Commedia. Il prof. Vilela infatti dichiara apertamente di propendere per le traduzioni- interpretazioni, come quella di Marques Braga e di Xavier Pinheiro, piuttosto che per quella di Graça Moura, il quale compie uno sforzo e a volte anche delle forzature nella lingua portoghese,per riuscire il più possibile a restare fedele al testo di Dante. Sembra questa una condotta condannabile dal nostro professore dell' Univercidade do Porto, perché una traduzione di questo tipo sarebbe, secondo lui, soltanto leggibile agli specialisti. Dichiara infatti l' autore del saggio che spesso ha avuto bisogno di tornare al testo italiano per capire il portoghese. D'altro canto, invece, dichiara che una traduzione come l'avrebbe consigliata Cicerone, ossia da orator e non da iterpres, 
è preferibile perché manterrebbe la forza e il colorito del testo di origine. Ora il prof. Vilela usa come fonti per avvalorare la sua tesi dei testi un po' datatui, da un lato il De optimo genere interpretandi di S. Girolamo, testo del 395 d. C, e dall'altro un appunto di Lutero sulla traduzione. Nel primo il santo, attenendosi fedelmente all'aucoritas ciceroniana, consiglia non una traduzione verbum pro verbo, ossia letterale, ma una che conservi il genus e la vis delle parole, ossia la caratterizzazione e la forza. Il fine dovrebbe essere, secondo Girolamo, “ captare nella propria lingua il senso originale del testo, traducendo le idee e non le parole". Altra fonte usata dal prof. Villa è Martin Lutero dei “ Sendbrief vom Dalmatchen”il quale, userebbe, ancora una volta ,secondo il professor Villa, uno stile oratorio nella traduzione, ossia interpretando il testo, piuttosto che tradurlo letteralmente.

Non credo che attualmente, a conforto di una buona traduzione si possano usare, da un lato una fonte medievale e, dall'altra una rinascimentale. Credo che la prospettiva di una traduzione, che si proponga soprattutto dei fini didattici e quindi esegetici del testo, si debba preoccupare principalmente di attenersi il più possibile al testo di partenza, facendo coincidere, in un certo senso, le prospettive di una traduzione, con quelle di una edizione critica: vale a dire, malgrado il passaggio da un idioma ad un altro, mantenere intatto il più possibile, il corpus del testo di partenza, tenendo presente comunque, che un testo tradotto è sempre e comunque un altro testo, non l'originale. Nel caso la traduzione eminentemente letterale sia di difficile accesso a quanti non conoscano termini inusitati o storicamente obsoleti, anche nella lingua di arrivo, si potranno aggiungere delle note esplicative e attualizzanti. Questa sicuramente dovrebbe esser ritenuta una ottima strategia, non solo di traduzione, ma soprattutto didattica, dal momento che è necessario presentare, per una corretta interpretazione testuale, un testo tradotto che riproduca il più fedelmente possibile, il testo di partenza, anche se difficile.

Senza così fornire ulteriori commenti, lascio le tre storiche traduzioni citate dal prof. Vilela dal IX canto del paradiso vv. 109-114: 
Ma perché tutte le tue voglie piene

Ten porti che sien nate in questa spera

Proceder ancor oltre mi convene

Tu vuò saper chi è in questa lumera

Che qui aprresso me così scintilla

Come raggio di sol in acqua mera

La traduzione di Graça Moura:

Mas por todo o desejo que a ti vem

Saciar, que ca' nasceu nesta espera proceder inda devo mais além

Queres saber quem nesta chama espera

Que junto a mim agora assim cintila

Como raio de sol em água mera.

Quella di Marques Braga:

A fim de que sejam inteiramente satisfeitos todos os teus desejos Aparecidos aqui neste céu de Venus, devo juntar ainda algumas outras coisas Tu queres saber que alma esteja dentro da luz que cintila, ao meu lado, como um raio de Sol, na água limpa.

E per finire quella di Xvier Pinheiro Para ser a medida em todo cheia Dos teus desejos , nados n'esta esphera Do meu discurso inda prossegue a teia Ora queres saber a luz que era Que ali perto de mim tanto scintila Como o sol, que na lympha reverbera. 